\title{
El régimen de estabilidad jurídica de las inver- siones en Ecuador a partir de la expedición de la Ley Orgánica de Incentivos para Asociacio- nes Público-Privadas y la Inversión Extranjera ${ }^{1}$
}

\author{
Ricardo Montalvo Lara² \\ Investigador independiente
}

\begin{abstract}
Resumen
El presente artículo analiza el impacto que la expedición de la Ley Orgánica de Incentivos para Asociaciones Público-Privadas y la Inversión Extranjera ha tenido en el régimen de estabilidad de las inversiones en el derecho ecuatoriano, gobernado hasta antes de su expedición exclusivamente por el Código Orgánico de la Producción, Comercio e Inversiones. Asimismo, expone y hasta cierto punto busca aclarar algunas de las nociones más importantes que han provocado cambios significativos en el régimen de estabilidad, las cuales deben ser claramente entendidas tanto por el inversionista como por el Estado ecuatoriano.
\end{abstract}

\begin{abstract}
This article analyzes the impact that the Organic Law of Incentives for Public-Private Partnerships and Foreign Investment has had in the regime of investment stability in Ecuadorian law, ruled before solely by the Organic Code Production, Trade and Investment. It also exposes and seeks to clarify, to some extent, the most important notions that have caused significant changes in the stability regime, and which must be clearly understood by both the investor and the Ecuadorian State.
\end{abstract}

Palabras clave:Estabilidad / Inversiones / Incentivos tributarios / COPCI / Asociaciones público-privadas

\section{Key Words}

Stability / Investment / Tax incentives / COPCI / Public-Private Partnerships

Sumario: 1. Introducción 2. La estabilidad para las inversiones en el Código Orgánico de la Producción, Comercio e Inversiones 3. La estabilidad jurídica para las inver-

1 Artículo recibido el 17 de abril y aceptado el 17 de junio de 2016.

2 Socio del estudio jurídico ML Abogados. Analista legal de inversiones y asociaciones público-privadas en el Ministerio

Coordinador de la Producción, Empleo y Competitividad. Abogado Summa Cum Laude por la Universidad San Francisco de Quito. 
siones en la Ley Orgánica de Incentivos para las Asociaciones Público-Privadas y la Inversión Extranjera 3.1 Estabilidad tributaria sobre los incentivos de la Ley de Incentivos APP 4. Estabilidad jurídica en la Ley de Incentivos APP 5. Estabilidad jurídica en el COPCI 6. Resumen y conclusiones

\section{Introducción}

Una de las preocupaciones más grandes de los inversionistas extranjeros respecto de países en vías de desarrollo radica en los continuos e impredecibles cambios en las regulaciones que tienen lugar en estos últimos. ${ }^{4}$ En el Derecho de las Inversiones, para mitigar este riesgo, han nacido varias protecciones jurídicas tanto en el campo internacional como en el nacional. ${ }^{5}$ En el derecho local, los países han creado instrumentos específicos, como los contrato de inversión, en los que han incluido mecanismos de estabilización con el fin de conciliar dos necesidades: la del Estado, de promover la inversión, nacional y extranjera; y la del inversionista, de focalizar sus recursos y realizar gastos de inversión prolongada en el tiempo en territorios donde el riesgo político no sea alto. ${ }^{6}$

Como explican Dolzer y Schreurer, más allá del reconocimiento de derechos, tareas, riesgos y responsabilidades, los contratos de inversión tienen como objetivo determinar las reglas del juego al que las partes (el Estado y el inversionista) se han comprometido. ${ }^{7}$ Desde una perspectiva legal, las más complejas y difíciles preguntas por responder al respecto se refieren usualmente a la inclusión de cláusulas que regulen las conductas de las partes en caso de cambios políticos en el país que recepta la inversión.Estas cláusulas se denominan "de estabilidad", y constituyen una variante especial de la cláusula de elección de ley aplicable que por regla general se incluye en los contratos de inversión. Para Cotula:

\footnotetext{
4 Así lo expone el Banco Mundial en su reporte anual de inversión y riesgo político, de acuerdo al cual, en el 2013, una vez más, el incumplimiento de los contratos y el ámbito regulatorio son las preocupaciones más grandes de los inversionistas respecto de los países en vías de desarrollo. El 40\% de los encuestados en el informe manifestó que tuvo pérdidas financieras por la emisión de regulaciones adversas que afectaron el retorno de su inversión. MIGA, "World Investment and Political Risk", Sitio web MIGA, 2014, disponible en: http://www.miga.org/documents/ WIPR13.pdf, p. 41 (acceso: 7/04/2016).

5 R. Dolzer y C. Schreurer, consideran que, una vez que un Estado ha permitido una inversión extranjera, el inversionista está protegido por las reglas de la costumbre internacional, los tratados internacionales y regionales e instrumentos de derecho local como leyes específicas que regulen la materia de inversión y contratos de inversión con disposiciones protectoras determinadas. Dolzer, R.; Schreurer C., Principles of International Investment Law. New York: Oxford University Press. 2008, pp. 7-11. 6 El riesgo político es un factor muy importante a la hora de decidir si invertir o no en un país determinado. El Banco Mundial, en su reporte del año 2013, expone claramente los tipos de riesgo político de mayor preocupación para los inversionistas en países en vías de desarrollo, así como el impacto del riesgo político en el inversionista extranjero. Banco Mundial, Banco Asiático de Desarrollo, Banco Internamericano de Desarrollo, Public Private Partnerships Reference Guide: version 2.0 <http://api.ning.com/ files/Iumatxx-0jz3owSB05xZDkmWIE7GTVYA3cXwt4K4s3Uy0NtPPRgPWYO1LLWaTUqybQeTXIeuSYUxbPFWlysuyNI5 rL6b2Ms/PPPReferenceGuidev02Web.pdf>, 2014, p. 21.

7 Dolzer, R.; Schreurer, C, Principles of International, p. 73.
} 
Stabilization clauses aim to "stabilize" the terms and conditions of an investment project, thereby contributing to manage non-commercial (that is fiscal, regulatory) risk. They involve a commitment by the host government not to alter the regulatory framework governing the project, by legislation or any other means [... ]. [De esta forma] under the so-called "freezing clauses", the applicable domestic law is the one in force at the time the contract is concluded. ${ }^{8}$.

Hasta el 29 de diciembre de 2015, el régimen de estabilidad para las inversiones en el Ecuador se encontraba regulado exclusivamente por el Código Orgánico de la Producción, Comercio e Inversiones —en adelante, COPCI—y su Reglamento a la Estructura e Institucionalidad de Desarrollo Productivo, de la Inversión y de los Mecanismos e Instrumentos de Fomento Productivo. Sin embargo, en los últimos años, Ecuador fue uno de los países con menor registro de inversión extranjera directa de Latinoamérica. ${ }^{9}$ Esto generó la necesidad de crear mecanismos que atraigan la inversión al país, incluyendo reformas al alcance y objeto del régimen de estabilidad.

Es por ello que la Asamblea Nacional aprobó la Ley Orgánica de Incentivos para Asociaciones Público-Privadas y la Inversión Extranjera ${ }^{10}$ —en adelante, Ley de Incentivos APP-, la cual implementó fuertes incentivos tributarios para los proyectos que se desarrollen bajo esa modalidad, ${ }^{11}$ así como modificó, de una forma significativa, el alcance y el objeto de la estabilidad en materia de inversiones en determinados sectores de la economía nacional. Bajo este contexto, el objetivo del presente trabajo es hacer un breve análisis acerca del impacto que la Ley de Incentivos APP ha tenido en el régimen jurídico de estabilidad de las inversiones en el derecho ecuatoriano, así como exponer y hasta cierto punto aclarar algunas de las nociones más importantes que han provocado cambios significativos en el régimen.

8 Cotula, L., "Regulatory Taking, Stabilization, 2008, pp. 5-6.

9 La Conferencia de las Naciones Unidas sobre Comercio y Desarrollo (UNCTAD, por sus siglas en inglés), en su reporte de inversión mundial de 2015, agrupó a Ecuador dentro de los países de Latinoamérica que menos recibieron inversión extranjera en 2014, en un rango de entre el 100 y 1000 millones de dólares. Conferencia de las Naciones Unidas sobre Comercio y Desarrollo (2015). World Investment Report 2015 <http://unctad.org/en/PublicationsLibrary/wir2015_en.pdf>, 2015, p. 58 10 La Ley entró en vigencia mediante Registro Oficial Suplemento 652 de 18 de diciembre de 2015.

11 La Guía de Referencia de Asociaciones Público-Privadas, elaborada por los organismos multilaterales de inversión pública más importantes del mundo otorga el siguiente concepto de asociación public-privada: "[a] long-term contract between a private party and a government entity, for providing a public asset or service, in which the private party bears significant risk and management responsibility, and remuneration is linked to performance". Banco Mundial, Banco Asiático de Desarrollo, Banco Internamericano de Desarrollo, Public Private Partnerships, p. 14. Sobre esta base, la Ley de Incentivos APP define a las asociaciones público-privadas, en su artículo 8, de la siguiente manera: Art. 8.- De la Asociación Público-Privada. Se define por asociación público-privada la modalidad de gestión delegada por la que el Gobierno Central o los Gobiernos Autónomos Descentralizados encomiendan al gestor privado, la ejecución de un proyecto público específico y su financiamiento total o parcial, para la provisión de bienes, obras o servicios a cambio de una contraprestación por su inversión, riesgo y trabajo, de conformidad con los términos, condiciones, límites y más estipulaciones previstas en el contrato de gestión delegada. 
Para el efecto, este trabajo está estructurado de la siguiente manera. En primer lugar, se revisarán las disposiciones relativas derecho de estabilidad contenidas en el COPCI. En segundo lugar, se analizarán algunas disposiciones de la Ley de Incentivos APP, con el fin de determinar las implicaciones que su reciente entrada en vigencia ha traído en nuestro ordenamiento en cuanto al derecho de estabilidad. Finalmente, se expondrán las conclusiones a las que se ha llegado después de haber realizado el análisis previamente mencionado.

\section{La estabilidad para las inversiones en el Código Orgánico de la Producción, Comercio e Inversiones}

El cuerpo legal más importante en materia de inversiones en Ecuador es el COPCI, el cual contiene un libro destinado exclusivamente al tratamiento del desarrollo de la inversión productiva y de sus instrumentos. Las normas de este libro reconocen garantías e incentivos a favor del inversionista privado, permitiendo al Estado, en todos sus niveles, otorgar tratamientos diferenciados, en calidad de incentivos, a favor de inversiones nuevas y productivas en función de sectores económicos, ubicación geográfica, entre otros factores. ${ }^{12}$

Sobre esta base, en el mismo COPCI se establecieron incentivos de orden tributario, reconocidos en su artículo 24, e incorporados como reformas a las normas tributarias correspondientes, ${ }^{13}$ como lo son la Ley Orgánica de Régimen Tributario Interno y la Ley Reformatoria para la Equidad Tributaria.

Con el fin de proteger estas inversiones y, sobre todo, estabilizar la normativa relativa a los incentivos tributarios, el COPCI contempló una herramienta jurídica a favor del sector privado: el contrato de inversión. Del artículo 25 del COPCI $^{14}$ podemos

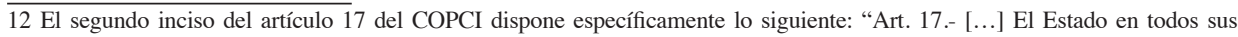
niveles de gobierno, en ejercicio de su plena potestad pública podrá otorgar tratamientos diferenciados, en calidad de incentivos, a favor de la inversión productiva y nueva, los que serán otorgados en función de sectores, ubicación geográfica u otros parámetros que éstas deberán cumplir, según los términos previstos en este Código y su Reglamento".

13 Así lo dispone el artículo 23 del COPCI: "Art. 23.- De los incentivos.- Los incentivos de orden tributario que reconoce esta normativa se incorporan como reformas a las normas tributarias pertinentes, como consta en las disposiciones reformatorias al final de este Código".

14 El artículo 25 del COPCI prescribe: Art. 25.- Del contenido de los contratos de inversión.- Por iniciativa del inversionista, se podrá suscribir contratos de inversión los mismos que se celebrarán mediante escritura pública, en la que se hará constar el tratamiento que se le otorga a la inversión bajo el ámbito de este Código y su Reglamento. Se podrá establecer en los contratos de inversión, los compromisos contractuales que sean necesarios para el desarrollo de la nueva inversión, los mismos que serán previamente aprobados por el ente rector de la materia en que se desarrolle la inversión. Los contratos de inversión podrán otorgar estabilidad sobre los incentivos tributarios, en el tiempo de vigencia de los contratos, de acuerdo a las prerrogativas de este Código. De igual manera, detallarán los mecanismos de supervisión y regulación para el cumplimiento de los parámetros de inversión previstos en cada proyecto. El Consejo Sectorial de la Producción establecerá los parámetros que deberán cumplir las inversiones que soliciten someterse a este régimen.
} 
abstraer los siguientes elementos esenciales del contrato de inversión: se trata de un negocio jurídico bilateral y solemne del cual nacen obligaciones para el Estado y los inversionistas, que son quienes lo suscriben. Por un lado, el Estado contrae dos obligaciones de hacer y una de no hacer. Las primeras consisten en reconocer los derechos y beneficios que el Código y su Reglamento le proveen a la inversión, y adquirir los compromisos contractuales que sean necesarios para el desarrollo de la nueva inversión. Por su parte, la de no hacer radica en no modificar para el inversionista las normas que se hayan declarado como invariables, lo que se traduce en otorgar estabilidad al inversionista, durante la vigencia del contrato, sobre los incentivos tributarios prescritos en el COPCI .

Mientras tanto, el inversionista se obliga a cumplir los parámetros de inversión establecidos por el Consejo Sectorial de la Producción. ${ }^{15}$ Este determinó como criterios objetivos de cumplimiento para el inversionista un mínimo monto de inversión, ${ }^{16}$ generación de empleo ${ }^{17}$ y porcentaje de contenido nacional. ${ }^{18} \mathrm{Si}$ el proyecto de inversión nueva y productiva cumple dichos parámetros, el Consejo Sectorial aprueba la suscripción el contrato y, luego de la suscripción, los criterios objetivos se transforman en obligaciones para el inversionista.

El tiempo de vigencia de estos contratos, a tenor del artículo 26 del COPCI, es “[... ] de hasta quince (15) años a partir de la fecha de su celebración, y su vigencia no limitará la potestad del Estado de ejercer control y regulación a través de sus organismos competentes". No obstante, el mismo artículo prescribe que "[... ] a petición

15 El Reglamento de Inversiones establece la composición y atribuciones del Consejo Sectorial en sus artículos 2 y 3 . El numeral 9 del artículo 3 claramente prescribe como una atribución del Consejo "Art. 3.- [...] 9. Establecer los parámetros que deberán cumplir las inversiones que soliciten someterse a los términos de los contratos de inversión referidos en el artículo 25 del Código; [...]”. Estos parámetros fueron fijados en la Resolución No. CSP-2012-002.

16 Art. 4.- Monto de la inversión.- De conformidad con el Código Orgánico de la Producción, Comercio e Inversiones, el monto mínimo de la inversión para la firma de un contrato de inversión deberá ser equivalente a USD 250.000,00 durante el primer año, valor que representará un mínimo del 25\% del valor total de la inversión prevista. El porcentaje de incremento de la inversión, la modalidad y la periodicidad de la misma, serán fijados durante el proceso de aprobación del proyecto por parte de la Secretaría Técnica en acuerdo con la inversionista, quien asume el compromiso de cumplir con los parámetros fijados y acepta el someterse a los procesos de monitoreo establecido en el Código Orgánico de la Producción, Comercio e Inversiones y en su Reglamento.

17 Art. 5.- Incremento de empleo.- Igualmente para establecer este parámetro y determinar el aporte de una inversión al incremento del empleo, se tomará en cuenta el número promedio de empleados del sector, y el porcentaje promedio de incremento salarial en los tres años anteriores a la fecha de la inversión, de manera que el parámetro fijado refleje según el caso, el incremento en número de trabajadores, o el incremento en mejora de sus condiciones laborales.

18 Art. 6.- Contenido nacional.- Para el caso de inversiones realizadas en sectores de sustitución de importaciones, fijados en el Reglamento a la estructura e institucionalidad de desarrollo productivo, se establecerá de conformidad con el porcentaje establecido por el Ministerio de Industrias, quien fijará tal porcentaje para aprobación del Consejo Sectorial de la Producción. Para el caso de inversiones que no se realicen en sectores de sustitución de importaciones, la Secretaría Técnica podrá requerir el pronunciamiento previo de parte del Ministerio de Industrias y Productividad, a efectos de que se convalide de manera oficial el porcentaje de contenido nacional que ofrece el inversionista incrementar a través de su proyecto de inversión, que constituirá uno de los criterios a ser considerados por el Consejo Sectorial de la Producción para la aprobación del respectivo contrato de inversión. 
del inversionista, y siempre que el Consejo Sectorial de la Producción lo considere pertinente, según el tipo de inversión que se esté desarrollando, los contratos de inversión podrán prorrogarse por una sola vez, hasta por el mismo plazo originalmente concedido".

Los demás elementos del contrato de inversión, así como su procedimiento de suscripción, están debidamente desarrollados y regulados por el Reglamento a la Estructura e Institucionalidad de Desarrollo Productivo, de la Inversión y de los Mecanismos e Instrumentos de Fomento Productivo —en adelante, Reglamento de InversionesEn este Reglamento se puede observar que, en razón de cómo se forma la voluntad administrativa en la etapa precontractual y de cómo el Consejo Sectorial de la Producción ejerce sus atribuciones, el contrato de inversión es un contrato de naturaleza administrativa. Esto se debe a que cumple lo previsto en el artículo 75 del Estatuto del Régimen Jurídico Administrativo de la Función Ejecutiva, el cual prescribe que un contrato administrativo "[e]s todo acto o declaración multilateral o de voluntad común; productor de efectos jurídicos, entre dos o más personas, de las cuales una está en ejercicio de la función administrativa".

Asimismo, el Reglamento desarrolla el alcance de la estabilidad tributaria que recae sobre los incentivos tributarios, circunscribiéndola a lo siguiente:

Art. 32.- Estabilidad de los incentivos Tributarios.- Durante la vigencia de los contratos de inversión se mantendrán invariables para el inversionista, la empresa receptora y la inversión, las normas legales y reglamentarias y resoluciones generales del Servicio de Rentas Internas, relativas a la determinación de la renta gravable de los inversionistas y la tarifa aplicable a la empresa receptora o la inversión, según el caso, vigentes a la fecha de celebración del contrato. De esta manera, los incentivos tributarios otorgados se mantendrán inalterables por el periodo de vigencia del contrato, a no ser que proceda la revocatoria de los mismos o la renuncia voluntaria por parte del inversionista.

Así, es posible concluir que la estabilidad del contrato de inversión consiste en un derecho personal a favor del inversionista de tornar invariables las leyes, reglamentos y actos normativos del Servicio de Rentas Internas que se relacionen con la determinación del impuesto a la renta.

Este derecho vuelve inalterables los incentivos tributarios que estén vigentes a la fecha de suscripción del contrato, así como la tarifa aplicable de impuesto a la renta para la empresa receptora (en caso de haberla) y la misma inversión. Esto genera que 
los incentivos tributarios no se puedan modificar por otras vías que no sean la revocatoria de los incentivos o la renuncia voluntaria del derecho de estabilidad por parte del inversionista. Hasta el 29 de diciembre de 2014, el mencionado líneas arriba era el único alcance de la estabilidad que concedía el contrato de inversión. Sin embargo, con la promulgación de la Ley Orgánica de Incentivos a la Producción y Prevención del Fraude Fiscal se añadió un capítulo innumerado al COPCI después de su artículo 26, denominado "Incentivo de estabilidad tributaria en contratos de inversión".

En este capítulo se amplió el alcance de la estabilidad, tal como lo prescribe su artículo innumerado tercero:

Alcance de la estabilidad tributaria.- La estabilidad tributaria, se limitará:

a) Para efectos de impuesto a la renta, la estabilidad se extenderá sobre todas las normas que permiten determinar la base imponible y la cuantía del tributo a pagar, vigentes a la fecha de suscripción del contrato de inversión. No aplicará sobre normas referentes a facultades, procedimientos, métodos y deberes formales que la administración tributaria emplee y establezca para el control y el ejercicio de sus competencias.

b) La estabilidad tributaria podrá hacerse extensiva al impuesto a la salida de divisas y otros impuestos directos nacionales, exclusivamente respecto de las tarifas y exenciones de cada impuesto, vigentes a la fecha de suscripción del contrato de inversión.

c) En el caso de las sociedades que realicen inversiones para la explotación de minería metálica a mediana y gran escala, cuya producción se destine a la exportación, también podrán obtener estabilidad tributaria del impuesto al valor agregado, exclusivamente respecto de sus tarifas y exenciones.

Es importante recalcar que el acceso a este derecho más amplio de estabilidad jurídica fue restringido para las sociedades que realicen inversiones para la explotación de minería metálica a mediana y gran escala.

Para que otros sectores puedan acceder a este beneficio, el COPCI estableció como requisitos que el monto de inversión sea mayor a USD 100 millones, que el Ministerio con competencias en el ámbito de la inversión realice un informe técnico sobre los beneficios económicos que reportará dicha inversión para el país, y que el Comité de Política Tributaria emita una autorización para la suscripción del contrato. ${ }^{19}$

19 Art. (...).- Ámbito de aplicación.- Las sociedades que realicen inversiones para la explotación de minería metálica a mediana y gran escala, de acuerdo a las condiciones que se establecen en el presente capitulo, tendrán derecho a beneficiarse de la estabilidad 
En todo caso, sin perjuicio de la ampliación al alcance de la estabilidad que concedieron estas reformas a los contratos de inversión, la estabilidad continuó centrándose en el ámbito estrictamente tributario. Por ello, en virtud de estas disposiciones, cualquier otra norma que no se refiera a esa materia no podía ser invariable en el tiempo, permitiendo al Estado mantener su potestad regulatoria independientemente de las posibles afectaciones que su potestad podría producir en la inversión privada.

Finalmente, el derecho de estabilidad, independientemente de su alcance, al haber sido otorgado por el contrato de inversión, es exigible en caso de que el Estado incumpla su obligación de no hacer. El COPCI permite que todas las disputas relativas a los derechos y obligaciones del contrato de inversión se puedan someter a arbitraje internacional ${ }^{20}$ o nacional, ${ }^{21}$ siempre y cuando no se traten directamente disputas en materia tributaria, excluidas expresamente por la Ley como no arbitrables.

Dado que la estabilidad no es materia tributaria sino un derecho contractual coligado a una obligación por parte del Estado de mantener la normativa tributaria invariable para el inversionista durante la vigencia del contrato de inversión, está dentro del alcance ratione materiae que la Ley establece para los convenios arbitrales en contratos de inversión.

tributaria, durante el plazo de vigencia del contrato de inversión. Dicha estabilidad tributaria también podrá' ser concedida a petición de parte, en el contrato de inversión de las sociedades de otros sectores, incluyendo a las industrias básicas, que realicen inversiones productivas para el desarrollo del país, siempre que:

1. El monto de la inversión sea mayor a 100 millones de dólares;

2. Informe técnico realizado por el Ministerio con competencias en el ámbito de la inversión, sobre los beneficios económicos que reportará dicha inversión para el país; [Numeral 3 derogado por disposición reformatoria primera de Ley No. 0, publicada en Registro Oficial Suplemento 493 de 5 de Mayo del 2015].

4. Autorización del Comité de Política Tributaria para la suscripción de un contrato en el cual se regularán los requisitos, montos y plazos para realizar la inversión así como los compromisos asumidos por el inversor.

20 Art. 27.- Resolución de conflictos.- En los contratos de inversión con inversionistas extranjeros se podrán pactar cláusulas arbitrales para resolver las controversias que se presenten entre el Estado y los inversionistas. Las controversias entre un inversionista extranjero con el Estado ecuatoriano, que se hubieren agotado completamente por la vía administrativa, intentarán solucionarse de manera amistosa, con diálogos directos por un término de 60 días. Si no se llegase a una solución directa entre las partes deberá existir una instancia obligatoria de mediación dentro de los tres (3) meses siguientes a la fecha de inicio formal de las negociaciones directas. Si luego de esta instancia de mediación la controversia subsiste, el conflicto podrá ser sometido a arbitraje nacional o internacional, de conformidad con los Tratados vigentes, de los que Ecuador forma parte. Las decisiones de ese Tribunal Arbitral serán en derecho, la legislación aplicable será la ecuatoriana y los laudos serán definitivos y obligatorios para las partes. Si luego del término de 6 meses de agotada la vía administrativa, las partes no han llegado a un acuerdo amistoso, ni la hubieren sometido a jurisdicción arbitral para la solución de sus conflictos, la controversia se someterá a conocimiento de la justicia ordinaria nacional. No se someterán a arbitraje los asuntos tributarios.

21 Art. 27.- [...] En caso de que se pacte arbitraje nacional, este se ventilará en un Centro de Arbitraje de reconocida trayectoria, de no menos de diez años de existencia y notable experiencia. El arbitraje será en derecho y el Tribunal se conformará con tres árbitros. 


\section{La estabilidad jurídica para las inversiones en la Ley Orgánica de Incentivos para las Asociaciones Público-Privadas y la Inversión Extranjera}

\subsection{Estabilidad tributaria sobre los incentivos de la Ley de Incentivos APP}

La Ley de Incentivos APP nació con el objeto de establecer incentivos para la ejecución de proyectos bajo la modalidad de asociación público-privada ${ }^{22}$. Estos incentivos consisten en importantes exenciones tributarias para los proyectos que se ejecuten bajo esa modalidad, que recaen sobre el impuesto a la renta, el Impuesto a la Salida de Divisas (ISD) y los tributos al comercio exterior.

Por un lado, el incentivo de impuesto a la renta consiste en una exoneración de 10 años para las sociedades que se creen o estructuren para desarrollar proyectos bajo la modalidad de asociación público-privada, contados a partir del primer ejercicio fiscal en el que se generen ingresos operacionales. Esta exoneración se extiende a los dividendos o utilidades que dichas sociedades paguen a sus socios o beneficiarios, cualquiera sea su domicilio ${ }^{23}$.

Por otro lado, las exenciones al pago del ISD constituyen beneficios para las sociedades estructuradas para el desarrollo de proyectos bajo la modalidad de asociación público-privada, que realicen pagos al exterior, independientemente del domicilio del receptor del pago. Tales exenciones tienen lugar para pagos por concepto de adquisición de bienes y servicios, pagos a financistas incluido el capital, intereses y comisiones, distribución de dividendos y pagos por adquisición de acciones y derechos representativos en general que sean emitidos por la sociedad. Por último, la exención a los tributos al comercio exterior ${ }^{24}$ se otorga para las importaciones directamente

22 Art. 1.- Objeto. Esta Ley tiene por objeto establecer incentivos para la ejecución de proyectos bajo la modalidad de asociación público-privada y los lineamientos e institucionalidad para su aplicación.

23 El artículo 9.3 de la Ley Orgánica de Régimen Tributario Interno dispone exactamente lo siguiente: Art. 9.3.- Exoneración del impuesto a la renta en el desarrollo de proyectos públicos en asociación público-privada.- Las sociedades que se creen o estructuren en el Ecuador para el desarrollo de proyectos públicos en asociación público-privada (“APP”), gozarán de una exoneración del pago del impuesto a la renta durante el plazo de diez años contados a partir del primer ejercicio fiscal en el que se generen ingresos operacionales establecidos dentro del objeto de la APP, de conformidad con el plan económico financiero agregado al contrato de gestión delegada, siempre que el proyecto se realice en uno de los sectores priorizados por el Comité Interinstitucional de Asociaciones Público-Privadas y cumplan con los requisitos fijados en la ley que regula la aplicación de los incentivos de las APP. Están exentos del impuesto a la renta durante el plazo de diez años contados a partir del primer ejercicio fiscal en el que se generen los ingresos operacionales establecidos dentro del objeto de la APP, los dividendos o utilidades que las sociedades que se constituyan en el Ecuador para el desarrollo de proyectos públicos en APP, paguen a sus socios o beneficiarios, cualquiera sea su domicilio.

24 El artículo 125 del COPCI, prescribe lo siguiente a partir de la reforma en la Ley de Incentivos APP: “Art. 125.- Exenciones.[...] Las importaciones directamente destinadas para la ejecución de proyectos públicos ejecutados en la modalidad de asociación público-privada que efectúen los participantes privados responsables, de conformidad con los contratos de gestión delegada celebrados con el Estado y sus instituciones, gozarán de los mismos beneficios, sean de carácter tributario o de cualquier otra naturaleza, que goza la entidad pública delegante en sus importaciones, siempre que el monto total de importaciones se ajuste a 
destinadas para el desarrollo de proyectos públicos ejecutados bajo la modalidad de asociación público-privada, efectuadas no solo por la sociedad que se estructure para ejecutar el proyecto, sino por todos los participantes privados responsables, de conformidad con los contratos de gestión delegada. La norma incluso prescribe que los participantes gozan de los mismos beneficios, sean de carácter tributario, o de cualquier otra naturaleza, de los que goza la entidad pública delegante en sus importaciones, siempre que el monto total de importaciones se ajuste a los criterios determinados por el Comité Interinstitucional de Asociaciones Público-Privadas para cada sector priorizado.

Todas las exenciones descritas constituyen importantes beneficios para los inversionistas, pues les permiten disminuir significativamente los costos de sus proyectos. Sin embargo, a diferencia de lo que el COPCI hace con sus incentivos tributarios, la Ley de Incentivos APP no otorga expresamente estabilidad sobre estas exenciones.

En este sentido, dicha Ley únicamente prescribe, en su artículo 15, que sus incentivos "se mantendrán mientras el contrato de gestión delegada se encuentre vigente, salvo las exenciones previstas en el artículo 9.3 de la Ley Orgánica de Régimen Tributario Interno". El verbo "mantener" no es uno que se aplique usualmente para calificar a un incentivo tributario. Por lo tanto, es necesario determinar qué es lo que el "mantenimiento" significa para efectos de la Ley, y así concluir si se trata de un derecho de estabilidad sobre las normas o se trata simplemente de un plazo de vigencia de los incentivos.

De acuerdo al numeral 2 del artículo 18 del Código Civil, "[l]as palabras de la ley se entenderán en su sentido natural y obvio, según el uso general de las mismas palabras; pero cuando el legislador las haya definido expresamente para ciertas materias, se les dará en éstas su significado legal". En materia tributaria, no existe un significado legal de mantenimiento, por lo que es necesario acudir a su significado natural.

Según el Diccionario de la Real Academia Española, el verbo "mantener" significa "conservar algo en su ser, darle vigor y permanencia" y "amparar a alguien en la posesión o goce de algo" (Real Academia Española, 2014). Esta definición no da todas las luces para determinar si "mantenimiento" significa estabilidad o si más bien se circunscribe al concepto de vigencia.

los criterios determinados por el Comité Interinstitucional de Asociaciones Público-Privadas para cada sector priorizado. Para este propósito la entidad pública delegante expedirá a favor del participante privado responsable de las correspondientes importaciones un certificado que acredite el destino de los bienes a ser importados y los resultados de sus estudios de evaluación efectuados en la etapa precontractual respecto de la cantidad y calidad de los bienes a ser importados".. 
Para dilucidar esto, es necesario entonces utilizar las demás reglas de interpretación que el Código Civil contempla. Por un lado, el artículo 18, en su numeral 4, ordena que "[e]l contexto de la ley servirá para ilustrar el sentido de cada una de sus partes, de manera que haya entre todas ellas la debida correspondencia y armonía”. Utilizando esta lógica, la primera parte del inciso se refiere al mantenimiento de los incentivos hasta que el contrato de gestión delegada se encuentre vigente, mientras que la segunda parte establece una excepción a este mantenimiento para las exenciones del artículo 9.3 de la Ley Orgánica de Régimen Tributario Interno.

Si es que mantener significaría "estabilidad", aquello querría decir que las exenciones del artículo 9.3 no gozarían de este derecho, al ser una excepción que la Ley expresamente contempla. Aquello resultaría un poco contradictorio, pues se estaría excluyendo de este derecho a una de las exenciones más importantes, como lo es la del impuesto a la renta. En cambio, si el mantenimiento significa "vigencia", aquello querría decir que el plazo de la mayoría de las exenciones sería el mismo que el plazo del contrato, y las únicas excepciones respecto de esa temporalidad serían las exoneraciones de impuesto a la renta, que en efecto tiene un plazo fijo de 10 años de acuerdo a la Ley Orgánica de Régimen Tributario Interno.

La segunda interpretación resulta mucho más plausible y armónica, sobre todo porque una ley otorga un plazo de vigencia a la exención, y la otra, en busca de no generar una contradicción respecto de dicho plazo, produce una exclusión, dando un plazo en cambio a las demás exenciones que no tenían una vigencia determinada por parte de sus propias leyes. Por último, este criterio se refuerza al aplicar la regla de interpretación del numeral 1 del artículo 18. Esta regla permite, para interpretar una expresión oscura de la ley, recurrir a su intención o espíritu claramente manifestados en ella misma, o en la historia fidedigna de su establecimiento.

Al revisar la historia de aprobación de la Ley de Incentivos APP, específicamente el proyecto de ley que el Presidente de la República envió a la Asamblea Nacional el 25 de septiembre de 2015, encontramos que la redacción respecto de los incentivos tributarios era distinta. El artículo 5 del proyecto de ley, cuyo encabezado era "estabilidad jurídica", expresaba lo siguiente sobre el tema:

Los incentivos tributarios que se otorgan a través de esta ley a las sociedades a cargo de la ejecución de proyectos públicos bajo la modalidad de asociación público-privada se aplicarán durante el plazo de vigencia del contrato de gestión delegada con independencia de las modificaciones que en el futuro se efectúen en el ordenamiento jurídico. (El énfasis es mío) 
No obstante, esta redacción fue modificada en el texto final de la Ley. Resulta evidente entonces que la Asamblea Nacional, durante el procedimiento de aprobación, modificó un artículo que otorgaba vigencia a los incentivos, independientemente de las modificaciones que se efectúen en el ordenamiento jurídico, por otro que simplemente se refiera a un mantenimiento de ellos. Así las cosas, resulta plenamente viable pensar que el cambio en la ley tuvo lugar para no otorgar estabilidad sobre los incentivos sino simplemente para definir su plazo de vigencia.

Este cambio, en todo caso, no parece generar una modificación sustancial al objetivo de que los incentivos se mantengan invariables en el tiempo a favor del gestor privado de la asociación público-privada. Si bien el artículo 16 de la Ley de Incentivos APP se refiere a la vigencia de los incentivos y no otorga per se un derecho de estabilidad sobre ellos, el hecho de que todos los incentivos constituyan exenciones y se sujeten a un plazo, permite, de forma abstracta, la misma invariabilidad.

Esto se debe a la naturaleza inmodificable que el derecho tributario les otorga a las exenciones cuya vigencia se sujeta a un plazo. Al respecto, Héctor Villegas afirma que:

[L]as exenciones respecto de las cuales la ley nada dice sobre término de vigencia se entienden otorgadas por tiempo indeterminado y subsisten mientras perduren los extremos fácticos tenidos en cuenta para concederlas.

La subsistencia de las exenciones esta también condicionada a que no sean derogadas las disposiciones legales que las establecen. O sea que el hecho de no expresarse término de vigencia no puede llevar a interpretar que la exención se otorgó "a perpetuidad" y una ley posterior puede suprimirla, cuestión, esta, que depende de la valoración del legislador de los intereses públicos en juego. [En cambio] Si se trata de un beneficio tributario concedido por un plazo determinado, dicho beneficio no puede ser legalmente abolido antes del vencimiento del plazo (el énfasis es mío). ${ }^{25}$

Nuestro Código Tributario recoge esta misma lógica, al prescribir en el artículo 34:

Art. 34.- Derogatoria o modificación.- La exención, aun cuando hubiere sido concedida en atención a determinadas situaciones de hecho, podrá ser modificada o derogada por ley posterior.

Sin embargo, la concedida por determinado plazo, subsistirá hasta su expiración. 
De lo expuesto se colige que la regla general es que las exenciones subsistan mientras no sean modificadas o derogadas. No obstante, si se sujetan a un plazo, se vuelven invariables desde que el plazo empiece a discurrir hasta su vencimiento.

En el caso de los incentivos tributarios de la Ley de Incentivos APP, al haberse sujetado todos ellos a un plazo, sea a través de la misma norma que otorga la exención o a través de otro cuerpo legal, es aplicable lo dispuesto en el segundo inciso del artículo 34 del Código Tributario. De esta forma, el derecho de exención subsiste hasta el vencimiento del plazo, sea de 10 años o de la duración del contrato, dependiendo del incentivo, y no puede ser derogado ni reformado; el efecto es muy similar al de una estabilidad tributaria durante el plazo de vigencia del contrato. Esto genera un significativo cambio respecto del tiempo de invariabilidad de los incentivos: el COPCI otorga hasta 15 años de estabilidad, mientras que la Ley de Incentivos APP lo hace por todo el tiempo que el contrato esté vigente (salvo un par de excepciones).

Esta distinción, asimismo, produce un impacto importante en el ámbito de resolución de controversias. El artículo 20 de la Ley de Incentivos APP, siguiendo la misma lógica del COPCI, prohíbe, en su tercer inciso, el sometimiento a arbitraje de los asuntos que se relacionen con materia tributaria. ${ }^{26} \mathrm{Al}$ tratarse de la vigencia de una exención, el cual es un tema directamente relacionado con esta rama del derecho, no es posible someterla a este método alternativo de resolución de conflictos. Consecuentemente, en caso de que se modifique o derogue uno de los incentivos y se aplique tal modificación o derogación en contra de un gestor privado que goce de ellos, tal desavenencia debería ventilarse por la vía contencioso-tributaria.

Aquello generaría un gran desincentivo para los inversionistas, principalmente los extranjeros, pues no podrían sujetar a un foro neutral los conflictos derivados de la modificación o derogación de los incentivos, los cuales de forma adicional, y particularmente en materia de asociaciones público-privadas, involucran muy grandes montos de inversión. Como afirman Dolzer y Scheuer:

In the absence of an agreement to the contrary an investment dispute between a state and a foreign investor would normally have to be settled by the host state's courts. [... F From the investor's perspective, this is not an attractive solution. Rightly or wrongly, the investor will fear a lack of impartiality from the courts of the state against whom it wishes to pursue its claim. In many countries an independent judiciary cannot be taken for granted and executive interventions in court proceedings or a sense of judicial loyalty

26 Art. 20.- [...] No se someterán a arbitraje los asuntos tributarios, así como ningún otro acto que se derive directamente de la potestad legislativa y regulatoria del Estado ecuatoriano. 
to the forum state are likely to influence the outcome of proceedings. This is particularly so where large amounts of money are involved. [...] Also, the ordinary courts may lack the expertise to deal with the sometimes highly technical questions of international investment law. ${ }^{27}$

De igual forma, tornaría mucho menos eficiente el impacto de esta particular estabilidad, provocando que no se cumpla el objetivo de mitigar el temor de los inversionistas ante la inseguridad respecto de las reglas del juego en el país.

Afortunadamente, para subsanar esta dificultad, la Ley de Incentivos APP contempló disposiciones reformatorias que permiten la suscripción de un contrato de inversión que otorgue un derecho de estabilidad sobre los incentivos tributarios otorgados en ella y permita la ventilación de desavenencias derivadas del ejercicio de dicho derecho por la vía arbitral. El numeral 2 de la Disposición Reformatoria Primera incluyó, después del número 3 del artículo 24 del COPCI, un último numeral a través del cual se reconoce, como incentivos tributarios para inversiones nuevas y productivas, los incentivos otorgados por la Ley Orgánica de incentivos para asociaciones público privadas:

4. Para proyectos públicos ejecutados en asociación público-privada: Las inversiones que se realicen en el contexto de la ejecución de proyectos públicos en la modalidad de asociación público-privada podrán obtener las exenciones al impuesto a la renta, al impuesto a la salida de divisas, a los tributos al comercio exterior y más beneficios previstos en la Ley de Régimen Tributario Interno para este tipo de proyectos públicos de conformidad con los requisitos previstos en los capítulos II y III de la Ley Orgánica de Incentivos para Asociaciones Público-Privadas y la Inversión Extranjera.

Esto permitió que el contrato de inversión, cumpliendo su objeto de otorgar estabilidad sobre los incentivos tributarios del artículo 24 del COPCI, pueda tornar invariables todas las normas tributarias relativas a los incentivos de la Ley de Incentivos APP, con el alcance determinado en el artículo 32 del Reglamento de Inversiones, durante el tiempo de vigencia de los contratos.

Para permitir que el derecho de estabilidad pueda extenderse durante toda la vigencia del contrato de gestión delegada, la Ley de Incentivos APP reformó el artículo 26 del COPCI, prescribiendo que "[l]os contratos de inversión celebrados con ocasión del desarrollo de un proyecto público en asociación público-privada tendrán la misma vigencia del respectivo contrato de gestión delegada". En este sentido, la norma 
incluso dispone, manteniendo el concepto de estrecho relacionamiento entre un contrato y otro, que "[l]a terminación del contrato de gestión delegada conlleva así mismo la del contrato de inversión sin que se requiera declaración o trámite adicional".

Adicionalmente, al suscribir el contrato de inversión, el inversionista puede, ahora sí, acceder a arbitraje nacional o internacional en caso de que se modifiquen o deroguen sus incentivos tributarios gracias al derecho de estabilidad ganado a través del contrato.

Como afirma César Zumárraga:

Si bien la medida soberana que en materia de tributos aplique al Estado solo puede ser, en sí misma, impugnada ante los jueces contenciosos administrativos [específicamente tributarios], sí es posible someter a arbitraje tales decisiones en cuanto a sus efectos y a la reparación de los perjuicios consiguientes, en la medida en que infrinjan derechos reconocidos al inversionista en los contratos con el Estado (v.gr. contrato de explotación, contrato de inversión u otros), que usualmente protegen al inversionista contra el trato discriminatorio, el cambio de ley, las medidas confiscatorias o de efecto equivalente, entre otras. ${ }^{28}$

Así, se supera la limitación impuesta por la Ley de Incentivos APP, pues la materia que se sometería a arbitraje ya no sería tributaria sino un derecho contractual sobre una obligación de no hacer del Estado, y el contrato principal del convenio arbitral no sería el contrato de gestión delegada sino el contrato de inversión.

\section{Estabilidad jurídica en la Ley de Incentivos APP}

Además de la estabilidad de los incentivos tributarios, muy similar a la del COPCI y otorgada asimismo a través de contrato de inversión, el artículo 15 de la Ley de Incentivos APP concedió un nuevo espectro de estabilidad a las inversiones que se ejecuten bajo la modalidad de asociación público-privada:

Art. 15.- De la Estabilidad Jurídica del Contrato de Gestión Delegada. La estabilidad jurídica que se garantiza en esta Ley se extiende a los aspectos regulatorios sectoriales y específicos que hayan sido declarados como esenciales en los correspondientes contratos de gestión delegada.

28 Zumárraga, C. “Arbitraje sobre Potestades Públicas en el Derecho Minero Ecuatoriano”, En Revista Ecuatoriana de Arbitraje, Editorial Cevallos, Quito, 2012, p. 39. 
Los contratos de gestión delegada deberán incluir cláusulas obligatorias relativas a la caducidad, causales de terminación de los contratos y las demás determinadas por la Ley.

La estabilidad jurídica no recaerá sobre las normas declaradas inconstitucionales o ilegales por el tribunal competente, durante la vigencia de los contratos de gestión delegada.

Los contratos de gestión delegada deben estar en armonía con los derechos, garantías y deberes consagrados en la Constitución de la República y respetar los tratados internacionales ratificados por el Estado ecuatoriano. [El énfasis es mío].

Esto constituye un cambio fundamental por varias razones. En primer lugar, la estabilidad se extendió sobre aspectos sectoriales específicos relacionados con la inversión, y su nacimiento como derecho se sujetó a la condición de declarar dichas normas como esenciales en el contrato de gestión delegada. De esta forma, se modificó el alcance de la estabilidad en razón de la materia, pero se incluyó un alto elemento de discrecionalidad con la inclusión del concepto jurídico indeterminado de "esencialidad". Consecuentemente, si bien su alcance se amplió, la efectiva aplicación del derecho adquirió cierto grado de incertidumbre.

El segundo cambio trascendental radicó en que el contrato de inversión, según la Ley de Incentivos APP, dejó de ser la fuente de este derecho de estabilidad. De acuerdo al primer inciso del artículo 15 de la Ley de Incentivos APP, el contrato de gestión delegada constituye únicamente el medio para declarar esenciales las normas a estabilizarse. Esto genera una muy importante variación con el régimen anterior, dado que la fuente de la estabilidad, según el COPCI, era contractual y exigible bajo el supuesto del incumplimiento mientras el contrato se encontrara vigente, independientemente de modificaciones en la ley. En cambio, para la Ley de Incentivos APP, la fuente de la estabilidad jurídica es únicamente la ley, causando que el derecho sea exigible mientras esta se encuentre vigente y no sea modificada.

Por último, el artículo determina en su tercer inciso que la estabilidad no puede recaer sobre normas declaradas como inconstitucionales o ilegales por un tribunal competente durante la vigencia del contrato de gestión delegada. Esto, en principio, parece ser una excepción a la estabilidad, negando su aplicación sobre normas que no sean válidas; sin embargo, leyéndolo detenidamente, constituye más bien una causal de pérdida de la misma en razón de la temporalidad que el inciso configura. 
La declaración de inconstitucionalidad o ilegalidad de la norma tiene lugar una vez que el contrato está en ejecución, mientras que las normas se declaran como esenciales cuando el contrato se suscribe. Por ello, lo que ocurre realmente no es que no se puede estabilizar la normativa, ya que la estabilidad tiene lugar en un momento anterior a la declaración, sino que una vez estabilizada, esta calidad se pierde en razón de la declaración de inconstitucionalidad o ilegalidad.

Esta causal de pérdida de estabilidad no estaba comprendida en las causales que el Reglamento de Inversiones establece para el contrato de inversión en su artículo 32, el cual estabiliza las normas independientemente de cualquier declaración judicial o administrativa que tuviera lugar de forma posterior.

\section{Estabilidad jurídica en el COPCI}

Finalmente, esta política expansiva del alcance de la estabilidad jurídica de la Ley de Incentivos APP reformó el COPCI, otorgando este derecho a otros sectores cuyas inversiones no se ejecuten necesariamente bajo la modalidad de asociación públicoprivada amparada por la Ley de Incentivos APP. La Disposición Reformatoria Sexta estableció lo siguiente:

\section{Luego del artículo 96 [del COPCI], agréguese el siguiente:}

Art. (....).- Estabilidad jurídica de la inversión.- Además de la estabilidad tributaria que se garantiza en este Código, se podrá otorgar estabilidad jurídica de la normativa sectorial específica que hubiese sido declarada como esencial en los correspondientes contratos de concesión u otros títulos habilitantes para la gestión de sectores estratégicos o la provisión de servicios públicos.

El plazo de vigencia de dicha estabilidad jurídica será el mismo plazo del contrato de inversión.

Así, la nueva estabilidad jurídica que se garantiza en el COPCI es muy similar a la de la Ley de Incentivos APP, pues también recae sobre aspectos sectoriales específicos relacionados con la inversión, y su nacimiento como derecho se sujeta a la condición de declarar dichas normas como esenciales en los contratos respectivos. No obstante, este derecho se otorga directamente para sectores estratégicos y para la provisión de servicios públicos, a diferencia de lo que ocurre con la Ley de Incentivos APP.

Esta última prescribe en su artículo 13 que, por excepción, el Comité Interinstitucional de Asociaciones Público-Privadas puede aprobar y priorizar el empleo de la 
modalidad de asociación público-privada para la prestación de servicios públicos. De igual forma, dispone que las disposiciones de la Ley no son aplicables para sectores estratégicos, excepto el sector hidroeléctrico y de energías alternativas. En este sentido se consigue, a través del COPCI, que la estabilidad jurídica rebase los límites de la asociación público-privada, y se aplique para toda delegación del Estado al privado en esos dos sectores.

Finalmente, la reforma liga la vigencia de la estabilidad jurídica a la del contrato de inversión, pues claramente dispone que el plazo de ambos sea el mismo. No es claro si, gracias a esta disposición, el contrato de inversión es solo un límite al plazo de la estabilidad jurídica, cuya fuente sería legal, o si más bien el contrato se transforma en la fuente de dicha estabilidad.

Si la fuente de la estabilidad fuera legal, se seguiría la misma lógica de la estabilidad jurídica del artículo 15 de la Ley de Incentivos APP, en cuanto a que este derecho es exigible mientras la ley esté vigente y no sea modificada. En cambio, si la fuente de la estabilidad es el contrato de inversión, esta es exigible bajo el supuesto del incumplimiento mientras el contrato se encuentre vigente, independientemente de modificaciones en la ley.

En todo caso, resulta evidente que el inversionista debe necesariamente celebrar un contrato de inversión para adquirir este beneficio. Para que la estabilidad pueda producir efectos, debe tener un plazo de vigencia, el cual está sujeto a la indefectible suscripción de un contrato de inversión, tanto si es que este es la fuente del derecho de estabilidad como si es que es un simple presupuesto para la determinación de su duración.

Sin perjuicio de lo expuesto, no cabe duda de que el efecto inmediato del numeral 6 de la Disposición Reformatoria Primera de la Ley de Incentivos APP es ampliar el espectro de la estabilidad jurídica, haciéndola la regla general para la delegación de servicios públicos, así como para los sectores estratégicos, expresamente excluidos por la Ley de Incentivos APP.

\section{Resumen y conclusiones}

El régimen general de inversiones en Ecuador estaba regulado exclusivamente por el COPCI, el cual otorga, por regla general, un derecho de estabilidad a favor del inversionista y de la inversión únicamente sobre sus incentivos tributarios, a través del contrato de inversión. Sin embargo, con la expedición de la Ley de Incentivos APP, este régimen cambió drásticamente. Se otorgó nuevos incentivos tributarios, basados exclusivamente en exenciones, a los cuales se otorgó un plazo determinado de 
vigencia. Al hacerlo, la naturaleza de la exención tributaria permitió que estos no puedan ser modificados o derogados por ley posterior mientras estén vigentes. Aquello significaba un gran aporte para el inversionista, quien en principio ya no necesitaba de un contrato de inversión para gozar de dicho derecho, así como la estabilidad se extendería por toda la duración del contrato de gestión delegada.

No obstante, la Ley excluyó la arbitrabilidad de materias tributarias en el ámbito de las asociaciones público-privadas, por lo que la posibilidad de someter a arbitraje las disputas relacionadas con la modificación o derogación de sus incentivos tributarios parecía imposible. Afortunadamente, y con el fin de otorgar una mayor seguridad jurídica a los inversionistas, la Ley de Incentivos APP reformó el COPCI, incluyendo a los incentivos APP como incentivos del artículo 24 de este último cuerpo legal. Así, permitió que el contrato de inversión pueda otorgar un derecho de estabilidad plenamente arbitrable al ya no tratarse de una materia tributaria per se.

Adicionalmente, la Ley de Incentivos APP otorgó un nuevo alcance de la estabilidad jurídica para las inversiones que se realicen a través de la modalidad de asociación público-privada y para las delegaciones, en general, en sectores estratégicos y servicios públicos. La estabilidad esta vez recaería sobre todas las regulaciones sectoriales y específicas que hayan sido declaradas como esenciales en los respectivos contratos, y la fuente de su origen sería directamente la Ley (al menos, con seguridad, en el caso de la estabilidad jurídica para las asociaciones público-privadas).

Es posible concluir, por consiguiente, que la Ley de Incentivos APP y sus reformas han generado un significativo incremento en el alcance de estabilidad en materia de inversiones en el Ecuador. Así, la Ley constituye una nueva herramienta para mitigar el riesgo político regulatorio que reina en los países latinoamericanos, lo cual representa una de las principales razones para limitar la atracción de inversión extranjera en estos países. Si bien esta no es la única ni la última forma para generar una mayor atracción de la inversión, sí constituye una muestra de interés por parte del país para obtener participación e inversión del sector privado en sectores en los que se le delegue a este último la gestión de actividades que están bajo la competencia del Estado. 


\section{Bibliografía}

Banco Mundial, Banco Asiático de Desarrollo, Banco Internamericano de Desarrollo, Public Private Partnerships Reference Guide: version 2.0 , 2014, http://api. ning.com/files/Iumatxx0jz3owSB05xZDkmWIE7GTVYA3cXwt4K4s3Uy0NtPPRgPWYO1lLrWaTUqybQeTXIeuSYUxbPFWlysuyNI5rL6b2Ms/PPPReferenceGuidev02Web.pdf, (acceso: 01/02/2016).

Conferencia de las Naciones Unidas sobre Comercio y Desarrollo, UNCTAD, World Investment Report, http://unctad.org/en/PublicationsLibrary/wir2015_en.pdf, 2015 (acceso: 03/02/2016).

Cotula, L., "Regulatory Taking, Stabilization Clauses and Sustainable Development", en: OECD Global Forum on International Investment VII "Best practices in promoting investment for development", París, 2008.

Dolzer, R.; Schreurer, C, Principles of International Investment Law, Oxford University Press, New York, 2008.

Multilateral Investment Guarantee Agency / MIGA, World Investment and Political Risk http://www.miga.org/documents/WIPR13.pdf, (acceso: 03/02/2016).

Real Academia Española, Diccionario de la lengua española, 23. a edición, Espasa, Madrid, 2014.

Villegas, $\mathrm{H}$, Curso de finanzas, derecho financiero y tributario, $7^{\text {a }}$ edición, Ediciones Depalma, Buenos Aires, 2001.

Zumárraga, C "Arbitraje sobre Potestades Públicas en el Derecho Minero Ecuatoriano", Revista Ecuatoriana de Arbitraje, Editorial Cevallos, Quito, 2012, pp. 1540.

\section{Legislación}

Código Orgánico de la Producción, Comercio e Inversiones / COPCI, http://www.proecuador.gob.ec/wp-content/uploads/2014/02/1-Codigo-Organico-de-la-Produccion-Comercio-e-Inversiones-pag-37.pdf, 2010 (acceso: 25/02/2016).

Reglamento a la estructura e institucionalidad del Desarrollo Productivo, de la inversión y de los mecanismos e instrumentos de fomento productivo establecidos en el Código Orgánico de la Producción, Comercio e Inversiones del Ecuador http://www.industrias.gob.ec/wp-content/uploads/downloads/2013/10/decre- 
to-757.pdf, 2011 (acceso: 28/03/2016).

Ley Orgánica de incentivos para asociaciones público privadas, http://www.industrias. gob.ec/wp-content/uploads/2016/04/LEY-ORGANICA-DE-INCENTIVOS-PARA-ASOCIACIONES-PUBLICAS-Y-PRIVADAS.pdf, 2015 (acceso: 26/03/2016). 